\title{
Blinded Clinical Study
}

National Cancer Institute

\section{Source}

National Cancer Institute. Blinded Clinical Study. NCI Thesaurus. Code C70840.

A clinical study that is designed using a procedure in which one or more parties to the study [subject(s), investigator(s), monitor, or/and data analyst(s)] are kept unaware of the treatment assignment(s). 